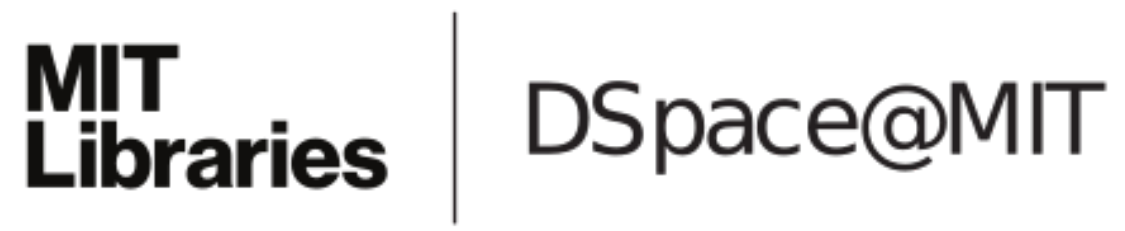

\author{
MIT Open Access Articles
}

\section{Flexible Reconfiguration of Existing Urban Water Infrastructure Systems}

The MIT Faculty has made this article openly available. Please share how this access benefits you. Your story matters.

Citation: Perelman, Lina Sela et al. "Flexible Reconfiguration of Existing Urban Water Infrastructure Systems." Environmental Science \& Technology 49.22 (2015): 13378-13384.

As Published: http://dx.doi.org/10.1021/acs.est.5b03331

Publisher: American Chemical Society (ACS)

Persistent URL: http://hdl.handle.net/1721.1/108068

Version: Author's final manuscript: final author's manuscript post peer review, without publisher's formatting or copy editing

Terms of Use: Article is made available in accordance with the publisher's policy and may be subject to US copyright law. Please refer to the publisher's site for terms of use. 


\title{
Flexible reconfiguration of existing urban water infrastructure systems
}

\author{
Lina Sela Perelman, ${ }^{*, \dagger}, \S$ Michael Allen, ${ }^{\ddagger}, \|$ Ami Preis, $₫$ Mudasser lqbal, ${ }^{\Uparrow}$ and \\ Andrew J. Whittle ${ }^{\dagger, \perp}$ \\ Department of Civil and Environmental Engineering, MIT, Cambridge, MA, USA, Faculty \\ of Engineering and Computing, Coventry University, UK, and Visenti ltd., Singapore \\ E-mail: linasela@mit.edu
}

\footnotetext{
${ }^{*}$ To whom correspondence should be addressed

${ }^{\dagger} \mathrm{MIT}$

${ }^{\ddagger}$ Coventry University

IVisenti

${ }^{\S}$ Postdoctoral Fellow

"Research Fellow

${ }^{\perp}$ Edmund K. Turner Professor
} 
and reconfigured systems is evaluated through direct and surrogate measures. The methodology is demonstrated using two large-scale water distribution systems showing the flexibility of our approach. The results highlight the benefits and disadvantages from network decentralization.

\section{Introduction}

Non-revenue water loss is the difference between the volume of water distributed through the system and the authorized/billed water consumption. Water losses include both real losses due to leaks in the pipes and apparent losses due to meter inaccuracy and unauthorized uses ${ }^{1}$. Water losses in distribution systems constitute a major inefficiency in water supplies due to wastage of treated water and energy resources, increases in operating costs, and reductions in revenue.

District metered areas (DMAs) are a cost-effective technology that has proven highly successful for water loss control and leakage management ${ }^{2,3}$. A DMA is a precisely defined sub-network, in which the inter-connecting pipes are monitored and the quantities of water entering and leaving the district are metered (enabling a better detectability of water losses through night flow diagnostics $)^{4}$. In addition, pressure management is aided by installing pressure reducing valves (PRV) at the inlet of each $\mathrm{DMA}^{5-7}$. The control of pressures in each DMA leads to a reduction in leakage through pipe joints and connections. DMAs were first introduced in the UK water industry in the early 1980 and have been reported to achieve a $85 \%$ reduction in measured leakage ${ }^{3,8}$. From water security perspective, some studies have suggested that in the event of a large scale contamination incident, the DMA structure would limit the spread of contamination and minimize the extent of response actions required for the system to restore to its normal pre-event conditions. The principal criteria of a DMA design are: (i) connectedness to the water source, (ii) size limits for each sub-network, (iii) minimum number of inter-connections, (iv) independence of the sub-networks, (v) minimum investment for the installation of isolation valves, and (vi) conserving system performance. 
The design of DMAs results in a star-like topology of the water distribution network comprising independent sub-systems that directly or through transmission mains are connected to water sources.

A number of methods for reconfiguration of water systems into DMAs have been previously suggested. These vary from manual trial and error approaches ${ }^{9}$ to automated tools integrating network analysis ${ }^{10}$, graph theory ${ }^{11-13}$, complex networks ${ }^{14,15}$, and heuristic methods ${ }^{15,16}$. The common workflow for DMA design is to identify water mains, partition the network into sub-networks, and isolate inter-connecting lines using simulation-based heuristics to minimize the number of connections and dependencies between the sub-networks. Table 1 in the Supporting Information (SI) presents a non-exhaustive list of recent research related to DMA design and their key features. The main drawbacks the prior methods for DMA design are that all of the studies link heuristic-based approaches with external simulation tool (e.g., EPANET ${ }^{17}$, WDNetXL ${ }^{18}$ ), which are typically time consuming especially for large-scale water systems, and none of the works consider the location of existing valves assuming that any pipe in the system can be uniquely isolated, which is impractical for real application.

Our work contributes to previous works by: (i) allowing only existing valves to be closed, thus avoiding capital costs for installation of additional valves, (ii) approximating the network flow and link isolation as linear programming (LP) problem, which can be efficiently solved for large-scale systems using standard solvers (e.g. MOSEK ${ }^{19}$, Gurobi ${ }^{20}$ ), and (iii) performing a rigorous analysis of network performance and resiliency using a suite of direct and surrogate measures. The methodology is applied and demonstrated using two large-scale water networks that, although supply similar daily demand, exhibit different topological properties. 


\section{Methods}

In our approach for automated network reconfiguration into sub-networks, the control variables are the existing valves that can be closed, the input parameters are the diameter and flow thresholds for identifying the mains, the lower and upper bounds for identifying the subnetworks, and the minimum desired operating pressure at network nodes. The outcome of our approach is precisely defined sub-network structure achieved by closing a selected subset of valves, such that each sub-network has a minimum number of inter-connections, desired demand range, and its nodal pressures are above a desired minimum. Our approach consists of two main steps: (i) topology decomposition - the system is initially decomposed into the main and sub networks and (ii) optimization problem - the network flow and reconfiguration problem is approximated as a single-objective linear programming (LP) optimization problem. The feasibility of the resulting solution is validated by solving the full nonlinear flow model using EPANET ${ }^{17}$ hydraulic solver, and the performance of the original and the reconfigured network is evaluated and compared using direct and indirect measures.

\section{Network topology decomposition}

The topology of water distribution systems is composed of mixed branched and looped configurations. Transmission mains convey large flows from the water sources to distribution mains of the interior system and typically comprise larger diameter pipes. The distribution mains further distribute water to end consumers and typically comprise smaller diameter pipes $^{21}$. Network decomposition consists of two main phases: (i) identifying transmission mains and (ii) defining sub-networks, as described next.

\section{Transmission mains}

The primary step towards DMA configuration is to identify the connected subset of transmission mains (pipes, valves, pumps) that connect the water sources (reservoirs, tanks, wells) 
to the interior of the network. For real systems, classification of transmission mains based solely on pipes' diameters ${ }^{10}$ may be inadequate as these pipes may not be fully connected and smaller pipes may carry large volumes of flow as well. We identify transmission mains as the connected subset of links with diameters, $D$, and flows, $q$, higher than the specified thresholds, $D_{c}$ and $q_{c}$, respectively. Given a network graph $G$, a set of nodes $N$ consisting of source $N_{s}$ and demand $N_{d}$ nodes, and a set of links $E$ consisting of pipes $E_{p}$ and valves $E_{v}$ :

1. Find the subset of links, $E_{D} \subset E$, with diameters above a given threshold: $E_{D}=$ $\left\{(u, v) \in E \mid D(u, v) \geq D_{c}\right\}$.

2. Find the subset of links, $E_{F} \subset E$, with flows higher than a given threshold: $E_{F}=$ $\left\{(u, v) \in E \mid q(e) \geq q_{c}\right\}$, where $q$ can be computed by solving the full set on nonlinear flow equations ${ }^{22}$ or using hydraulic simulator ${ }^{17}$ :

3. Combine both sets, $E_{C}=\left\{E_{D} \cup E_{F}\right\}$, and find the largest connected component, $G_{\text {main }}$, in the subgraph $G\left(N_{C}, E_{C}\right)$ where $N_{C}=\left\{u \mid(u, v) \in E_{C}\right\}$, that is accessible from the sources. A connected component is a subgraph that contains a path between every pair of distinct nodes and can be found using the breadth first search (BFS) algorithm ${ }^{23}$ and setting each source node as the root node. Consequently, the subgraph $G_{\text {main }}$ is composed of the transmission mains and is connected to the sources.

4. Extend the connected subgraph of transmission mains such that it has only valves in its edge-cut. We define an edge-cut as the set of all links that have one node that belongs to a given subset of nodes $N_{i}$ and the other belongs to $N \backslash N_{i}$. Let $N_{\text {main }}=\left\{u \in N\left(G_{\text {main }}\right)\right\}$ be the set of all nodes in the main subgraph, $E_{\text {main }}=$ $\left\{(u, v) \in E\left(G_{\text {main }}\right) \mid u, v \in N_{\text {main }}\right\}$ be the set of all links in the main subgraph, and $E_{\text {cut-main }}=\left\{(u, v) \in E \backslash E_{\text {main }} \mid u \in N_{\text {main }}, v \in N \backslash N_{\text {main }}\right\}$ be the main edge-cut. Then the extended subgraph $\tilde{G}_{\text {main }}$ has only valves on its boundary connections and its edgecut contains only valves, with $\tilde{E}_{\text {cut-main }}=\left\{(u, v) \in E_{v} \mid u \in \tilde{N}_{\text {main }}, v \in N \backslash \tilde{N}_{\text {main }}\right\}$, $\tilde{N}_{\text {main }}=\left\{u \in N\left(\tilde{G}_{\text {main }}\right)\right\}, \tilde{E}_{\text {main }}=\left\{(u, v) \in E\left(\tilde{G}_{\text {main }}\right) \mid u, v \in \tilde{N}_{\text {main }}\right\}$. This is 
achieved by traversing network links in a BFS manner starting from each boundary node of the initial transmission main, $G_{\text {main }}$, and exploring all adjacent links until the closest existing valves are reached.

The outcome of the first step is the subgraph $\tilde{G}_{\text {main }}$ which is the center node of the startopology and will connect all sub-networks to the water sources.

\section{Graph decomposition}

The next step is to decompose the rest of the network, $G\left[N \backslash \tilde{N}_{\text {main }}\right]$, into sub-graphs such that each sub-graph is within a specified size range, has a connection to the source, has a minimum number of inter-connecting links, i.e. small edge-cut size, and all inter-connecting links are existing valves to avoid any additional retrofit costs. We treat inter-connecting valves as a hard constraint and the rest of the constraints as soft constraints, i.e. can be violated. We combine graph search and partitioning algorithms to decompose the water network, taking the following steps:

1. Identify all subgraphs $G_{i}$ connected to the main subgraph using BFS starting from each boundary node of $\tilde{G}_{\text {main }}$. Set counter $m=2$.

2. Compute the demand, $d\left(N_{i}\right)$, of each subgraph identified previously, where $N_{i}=N\left(G_{i}\right)$ is the set of nodes belonging to the subgraph, $G_{i}$. Given the minimum and maximum desired total demands, $\underline{d}$ and $\bar{d}$, respectively, check if:

$-d\left(N_{i}\right)<\underline{d} \Rightarrow$ merge small sub-networks with the main $\tilde{G}_{\text {main }}=\left\{\tilde{G}_{\text {main }} \cup G_{i}\right\}$

$-\underline{d}<d\left(N_{i}\right)<\bar{d} \Rightarrow$ create new sub-network $G_{m}=G_{i}, m=m+1$

- $d\left(N_{i}\right)>\bar{d} \Rightarrow$ further partition $G_{i}$ into $k$ subgraphs, using a graph partitioning algorithm (METIS ${ }^{24,25}$ ) with $k=\left\lfloor d\left(N_{i}\right) / \bar{d}\right\rfloor$. The graph partitioning algorithm is adopted from distributed computing for allocating tasks to multiple processors and it divides the given graph with $|N|$ nodes into $k$ clusters, such that the number of inter-connections between different clusters is minimized and the clusters 
are roughly the same size. This graph partitioning approach has bee previously successfully applied to water distribution systems ${ }^{26}$. Finally, as previously, each subgraph is refined to have only valves in its edge-cut.

The outcome of this step is a star-configuration of the water network based solely on topological properties, where $\tilde{G}_{\text {main }}$ and $G_{m}, m=1, \cdots, K$, are main and the sub-networks of the full water system and all inter-connections between the sub-networks are valves, $E_{\text {cut-m }}=\left\{(u, v) \in E_{v} \mid u \in N\left(G_{m}\right), v \in N \backslash N\left(G_{m}\right)\right\}$. Let $E_{\text {cut }-M} \subset E_{v}$ be the union of all valves in the edge-cut of each sub-network. Note, although in this application we focused on sub-network size in terms of demand, any function can be applied such as number of nodes or number of connections.

\section{Optimization problem formulation}

Next, we approximate the network reconfiguration as a linear programming (LP) problem, where the decision variables are the boundary valves in the edge-cut, the system is subject to hydraulic and operational constraints, and the objective function minimizes the number of open boundary valves.

\section{Network flow}

For each node $i \in N$ in the network, the conservation of water is written as:

$$
\sum_{k \in E_{i, \text { in }}} q_{k}-\sum_{k \in E_{i, \text { out }}} q_{k}=d_{i} \quad \forall i \in N
$$

where $q_{k}$ is the flow in link $k, E_{i, \text { in }}$ and $E_{i, \text { out }}$ are the links coming in and out of the node $i$, and $d_{i}$ is the nodal demand.

Then for each link $k \in E$ the conservation of hydraulic energy is written as:

$$
h_{k}+H_{j}-H_{i}=0 \quad \forall k \in E
$$


where $H_{i}, H_{j}$ are the hydraulic head at the start and end nodes $i, j \in N$, respectively, and $h_{k}$ is the head loss or gain of the hydraulic element. For network pipes, the headloss is a monotonically increasing power function of the flow rate that can be estimated using the Hazen-Williams model ${ }^{27}$ as:

$$
h_{k}=R_{k} q_{k}^{\alpha} \quad \forall k \in E_{p}
$$

where $R_{k}$ is the pipe's roughness coefficient, $\alpha=1.852$, and $E_{p}$ is the set of pipes. The headloss for valves follows the same power function (Eq. 3) with different parameters $R$ and $\alpha$ depending its characteristics.

The given network flow problem results in a set on nonlinear equations an embedding them into an optimization problem will result in a nonlinear nonconvex optimization problem. Several modeling and solution approaches have been suggested in past years exhibiting a clear trade-off between modeling complexity and efficiency of the solution approach. The main approaches rely either on some approximation of the flow model, such as linear relaxations ${ }^{28,29}$, which can then be efficiently solved using modern solvers, or solving the nonlinear models using heuristics or evolutionary algorithms ${ }^{16,30}$ but without solution guarantees. Additionally, the evolutionary algorithms tend to suffer from computational burden as the size of the optimization problem increases. To achieve a practical and efficient solution method we suggest a linear approximation of the nonlinear head loss function around an operating point taking the form:

$$
\tilde{h}_{k}=a_{1 k} q_{k}+a_{0 k} \quad \forall k \in E
$$

where $a_{1 k}, a_{0 k}$ are a function of selected operating point $q_{k}^{o p}, h_{k}^{o p}$ and $R_{k}$ pipe's characteristics, as shown in Figure 1 of the SI. Within the operating range, the linear model of a single pipe slightly overestimates the headloss. Outside the operating range with the flow in the same direction, the linear model underestimates the headloss, and significantly overestimates if the direction of flow changes. We later show, that we validate the feasibility of our final solution by solving the full set of nonlinear equations. 
Substituting Eq. (4) into Eq. (2), the approximated model of the hydraulic energy over network links takes the following form for all pipes and valves except the valves that are in the final edge-cut:

$$
a_{1 k} q_{k}+a_{0 k}+H_{j}-H_{i}=0 \quad \forall k \in E \backslash E_{c u t-M}
$$

For each boundary valve in the edge-cut $k \in E_{\text {cut-M }}$, that can be closed, we modify Eq. (5) to model zero flow. If the flow in the valves is zero, $q_{j}=0$, then according to Eq. (5), the head difference between the two previously adjacent nodes (before isolation) is strictly equal to $a_{0 k}$, which is obviously false. To model zero flow in isolated valves, for each valve, we introduce two additional variables $y_{k}, u_{k}$ and two additional constraints (6b-c) representing valve's state (open or closed) and the head difference between disconnected nodes in case of a closed valve. The set of new constraints is formulated as:

$$
\begin{aligned}
a_{1 k} q_{k}+a_{0 k}+H_{j}-H_{i}+u_{k}=0 & \forall k \in E_{\text {cut }-M} \\
\left(1-y_{k}\right) \underline{q_{k}} \leq q_{k} \leq \overline{q_{k}}\left(1-y_{k}\right) & \forall k \in E_{\text {cut-M }} \\
-M y_{k} \leq u_{k} \leq M y_{k} & \forall k \in E_{\text {cut-M }} \\
y_{k} \in\{0,1\}, u_{k} \in \mathbb{R} &
\end{aligned}
$$

where $u_{k}$ is a continuous variable representing head difference between disconnected nodes, $y_{k}$ is a binary variable representing the state of the valve ( 1 - closed, 0 - open), $M$ is a large number, and $E_{c u t-M}$ is the set of boundary valves.

The set of equations in (6) is reduced to two cases: (i) $y_{k}=1 \Rightarrow q_{k}=0, u_{k} \in \mathbb{R}$ - the valve is closed, the flow rate is zero, $q_{k}=0$, and the head difference between the two adjacent nodes is a real-valued number and (ii) $y_{k}=0 \Rightarrow q_{k} \in \mathbb{R}, u_{k}=0$ - the valve is open, the dummy variable $u_{k}$ is zero and Eq. (6a) preserves its original form as in Eq. (5). 


\section{Linear programming formulation}

Given a star-topology with inter-connecting valves, the problem is to find the largest subset of valves that can be closed such pressures are maintained above a desired minimum value. Combining Eqs. (1)-(6), the following LP problem is formulated:

$$
\begin{array}{cc}
\underset{\mathbf{q}, \mathbf{H}, \mathbf{y}, \mathbf{u}}{\operatorname{minimize}} & N_{v}-\sum_{k \in E_{c u t-M}} y_{k} \\
\text { subject to } & (1),(5),(6) \\
& \underline{H}_{i} \leq H_{i} \leq \bar{H}_{i} \quad \forall j \in N \\
& 0 \leq \mathbf{y} \leq 1
\end{array}
$$

where $N_{v}=\left|E_{\text {cut-M }}\right|$ is the number of boundary valves and $\underline{H}_{i}, \bar{H}_{i}$ are the lower and the upper pressure constraints, respectively. Note, that we relax the integer constraint and allow $y$ to vary between 1 and 0 , this is to capture the inaccuracies resulting from the linearization of the headloss function.

In the final solution, the valves corresponding $y=1$ are closed and the rest are left open and the feasibility of the solution is validated by solving the full set of nonlinear flow equations, e.g. using EPANET ${ }^{17}$.

\section{Performance evaluation}

Several measures have been previously suggested for analyzing the performance of water networks. These can be classified into direct measures of hydraulic reliability, e.g. minimum pressure and water age, surrogate physical metrics computed as a function of the energy dissipated in a system ${ }^{31}$, and complex networks indexes that analyze the structural robustness of water distribution networks ${ }^{32}$. Next, we briefly review the measures we use for analyzing network performance. 


\section{Direct measures}

1. Worst cut-size $(W C S)$ - is the largest edge-cut of an individual sub-network, $\left|E_{\text {cut-m }}\right|, m=$ $1, \cdots, K$. This measure indicates the maximum number of meters and control valves that are needed to control an individual sub-network and the extent of response actions in the event that the sub-network needs to be isolated.

2. Total cut-size $(T C S)$ - is the size of the edge-cut of the network $\left|E_{c u t-M}\right|$, i.e. the total number of boundary valves. This number indicates the overall investment required for network retrofit (flow meters and pressure control valves) and needs to be minimized.

3. Pressure - the performance of the system can be naturally evaluated based on the pressure distribution before and after reconfiguration.

4. Water age (WA) - water age is an indicator for water quality and is also used to evaluate network performance.

\section{Physical surrogate measures}

1. Resilience index ${ }^{33}-I_{R}$ is a measure of excess system power based on the power loss in a system and can be computed as:

$$
I_{R}=1-\frac{P_{\text {loss }}}{P_{\text {loss }}^{\text {max }}}=1-\frac{\sum_{i \in N_{s}} H_{i} d_{i}-\sum_{i \in N_{d}} H_{i} d_{i}}{\sum_{i \in N_{s}} H_{i} d_{i}}
$$

where $P_{\text {loss }}$ is the actual power loss in the network and $P_{\text {loss }}^{\max }$ is the maximum feasible power loss in the network. Higher values of the resilience index $I_{R}$ indicate a more efficient distribution of flows in term of power dissipation.

2. Network resilience index ${ }^{34}-I_{N}$ is a modified resilience index taking into account changes in pipe diameters:

$$
I_{N}=1-\frac{P_{\text {loss }}^{a d j}}{P_{\text {loss }}^{m a x}}=1-\frac{\sum_{i \in N_{s}} H_{i} d_{i}-\sum_{i \in N_{d}} U_{i} H_{i} d_{i}}{\sum_{i \in N_{s}} H_{i} d_{i}}
$$


where

$$
U_{i}=\frac{\sum_{k \sim(i, j)} D_{k}}{|k| \cdot \max \left\{D_{1}, \ldots D_{k}\right\}}
$$

where $P_{\text {loss }}^{a d j}$ is the modified actual power loss in the network adjusted to pipe diameters, $D$ is pipe diameter, $|k|$ is the number of pipes connected at node $i$, and $U_{i} \leq 1$ is a scale factor penalizing changes in diameters. Higher values of the network resilience index $I_{N}$ indicate a more efficient distribution of flows in terms of power dissipation and network design.

\section{Complex network measures}

1. Meshedness coefficient ${ }^{35}-R_{m}$ is defined as the fraction of the actual number of loops to the maximum possible number of loops in a planar graph: $R_{m}=(m-n+1) /(2 n-5)$, where $m$ is the number of links and $n$ is the number of nodes in the graph. This is a surrogate metric of path redundancy in a network.

2. Spectral gap ${ }^{36}-\Delta \lambda$ is the difference between first and second eigenvalues of graphs adjacency matrix $A$. A small spectral gap could indicate the presence of articulation points whose removal may split the network into isolated parts.

3. Algebraic connectivity ${ }^{37}-\lambda_{2}$ is the second smallest eigenvalue of normalized Laplacian matrix of the network. A larger value of algebraic connectivity denotes the network robustness and tolerance against efforts to decouple the network.

Network reconfiguration schemes and suggested performance analysis are demonstrated in Figure 2 in the SI using an illustrative example adopted from Alperovits and Shamir ${ }^{38}$.

\section{Applications and results}

The suggested approach was applied to two large-scale water networks - EXNet ${ }^{39}$ and BWS$\mathrm{NII}^{40}$. We randomly added valves to both networks to test our approach, as the original 


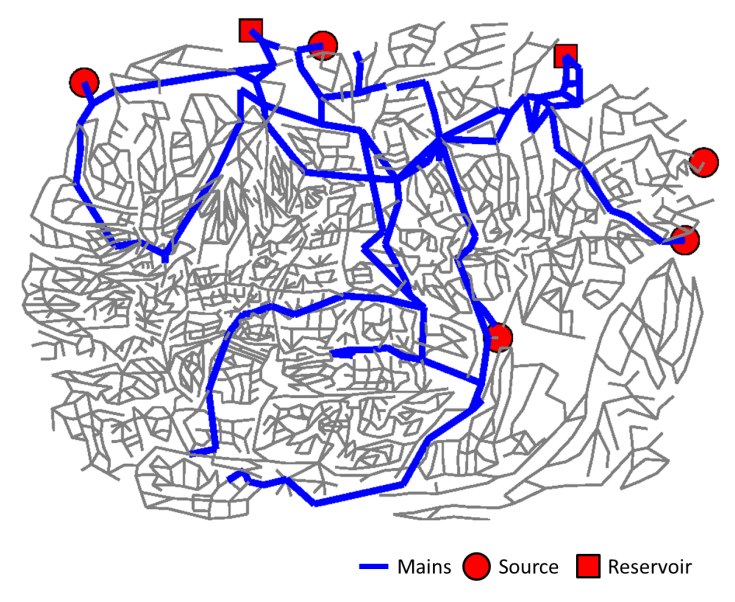

(a) Transmission mains

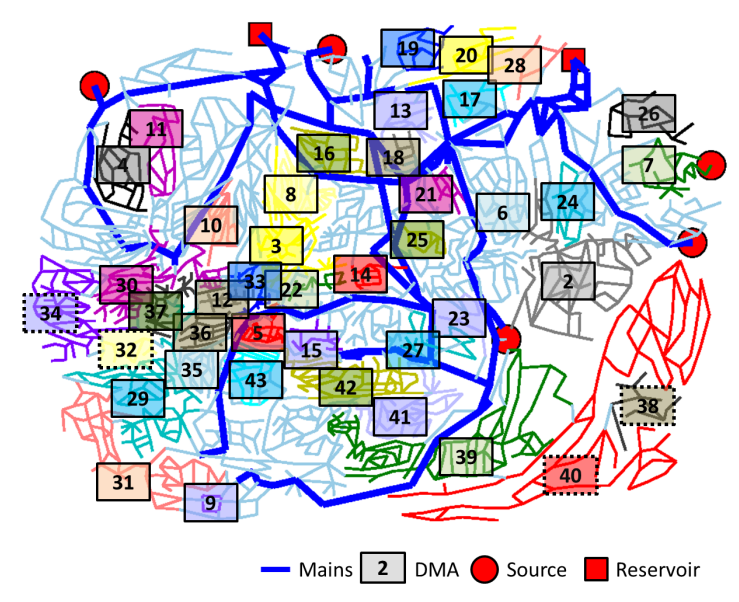

(b) Sub-networks

Figure 1: EXNet topology

Table 1: Data for water systems

\begin{tabular}{|c|c|c|c|c|c|c|c|}
\hline System & \#Pipes & \#Valves & \#Nodes & $\begin{array}{c}\text { Demand } \\
{\left[10^{6} \mathrm{gal} / \text { day }\right]}\end{array}$ & $\begin{array}{c}\text { \#sub } \\
\text { networks }\end{array}$ & $\begin{array}{c}\text { Full }^{*} \\
\text { cut-size }\end{array}$ & $\begin{array}{c}\text { Reduced }^{* *} \\
\text { cut-size }\end{array}$ \\
\hline EXNet & 1,546 & 872 & 1891 & 37 & 42 & 130 & 47 \\
\hline BWSNII & 11,024 & 3,295 & 12,523 & 28.2 & 36 & 206 & 49 \\
\hline
\end{tabular}

networks do not contain any valves, additionally, for the EXNet, we reduced the nodal demand by half since this network was developed for rehabilitation design to supply future demands. The complete EPANET ${ }^{17}$ files are available in the SI. The system data and design parameters used in this work are:

Network model. The required inputs include network topology, properties of network nodes and links (i.e., length, diameters, roughness of pipes, nodal elevations and daily demands). This information can also be read directly from the EPANET ${ }^{17}$.inp network files. Summary of networks' data is given in Table 1 (first five columns). The EXNet is a smaller network in terms of number of pipes and nodes, but it supplies slightly higher daily demand than BWSNII, which almost seven times larger in size.

Design parameters. For both networks, the demonstrated results are for the parameters: (i) threshold diameter $D_{c}=16[$ inch $]$ and threshold flow $q_{c}$ is the top $1 \%$ of network flows, (ii) minimum and maximum sub-network size $\underline{d}=10^{5}\left[\frac{\mathrm{gal}}{\mathrm{day}}\right]$ and $\bar{d}=10^{7}\left[\frac{\mathrm{gal}}{\mathrm{day}}\right]$, and (iii) minimum 
nodal pressure $\underline{P}_{i}=10[$ psi $]$, where the pressure head, $H_{i}$, is equal to the pressure plus the elevation of node $i$.

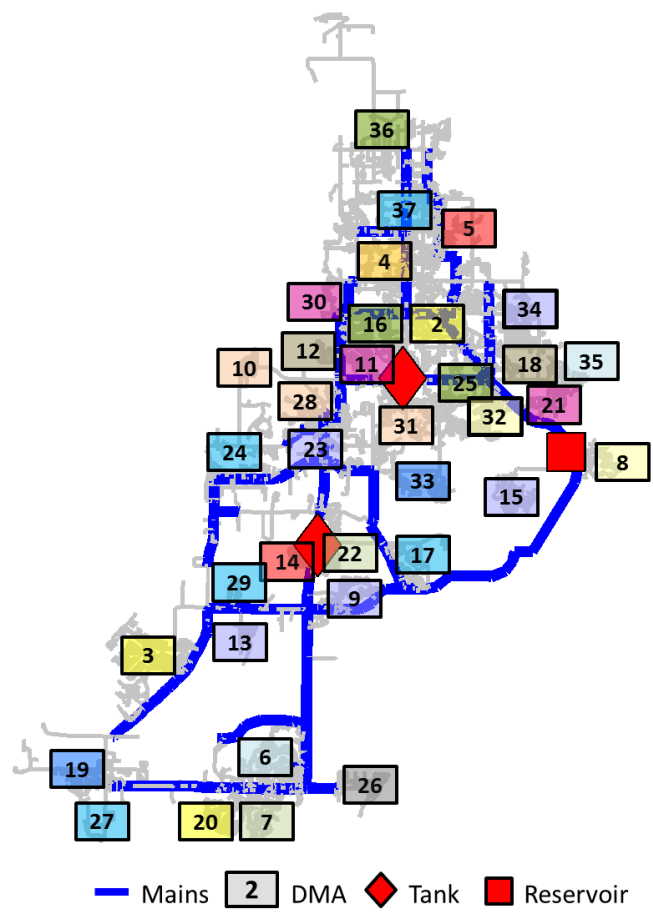

Figure 2: BWNSII mains and sub-networks topology

so

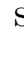
algorithm. Next, based on the graph decomposition steps described previously, the network was partitioned into 42 sub-networks with 130 boundary valves connecting the different subnetworks, as shown in Figure $1 \mathrm{~b}$ and listed in Table 1 in columns six and seven. Table 2 in the SI gives a detailed list of the demand, mean pressure, water age, and the size cut of each sub-network. All sub-networks are within the desired demand range and all, excluding $32,34,38$, and 40, which are located farther from the mains (shown in dashed line), have a direct connection to the transmission mains.

The BWSNII was partitioned into 36 sub-networks with 206 boundary valves. The layout of the BWSNII network, its transmission mains, and sub-networks are shown in Figure 2. As previously, all sub-networks are within the desired demand range and all have a direct 
connection to the transmission mains. Table 4 in the SI shows the demand, mean pressure, water age, and the size cut for each sub-network.

Optimization problem. We formulate the optimization problem based on (7). The EXNet has a single loading condition, hence for each pipe, we use two-point linear approximation with $\left[Q_{1} Q_{2}\right]=\left[\begin{array}{lll}0.5 q & 1.5 q\end{array}\right]$, where $q$ is the flow in each pipe. An example for the two-point linearization is given in Figure 1 of the SI. The LP model results in 4,569 decision variables and 4,829 constraints. The Gurobi solver ${ }^{20}$ is used to solve the optimization problem with a solution time around $0.6[\mathrm{sec}]$ (Intel Core i7 $2.9 \mathrm{GHz} 16 \mathrm{~GB}$ of RAM). The solution is the list of valves that can be isolated, i.e. with corresponding dummy variables equal to one, $y_{k}=1$. We refer to the full model as the network before closing valves and to the reduced model - after closing valves. For EXNet, 83 valves were identified for a potential isolation for network reconfiguration, with only 47 valves remaining open (Table 1, last column). As mentioned before, to validate the solution of the LP problem we solve the full set of nonlinear flow equations using EPANET ${ }^{17}$. All results demonstrated below are computed based on the hydraulic simulations using EPANET. The inp file of the reconfigured network can be found in the SI. A full list of the number of connections (cut-size) for each of the sub-networks before and after optimization is given in Table 2 of the SI and the detailed list of boundary valves at the solution is given in Table 3 of the SI.

For BWSNII we take the minimum and the maximum flows during the extended period simulation for the linear approximation of the headloss function and formulate the optimization problem for the peak demand condition. The LP model results in 16,521 decision variables and 16,538 constraints, with the solution time of approximately of 5.5[sec]. For BWSNII, 157 valves were closed, with only 49 remaining open (Table 1, last column). The solution was again validated using EPANET $^{17}$ simulations and the new .inp file can be found in the SI. The detailed lists are given in Tables 4 and 5 of the SI.

Performance evaluation. Next, we analyze the performance of the full and reduced models based on the different measures. Figure 3a shows the cut-size and Figure $3 \mathrm{~b}$ the average 


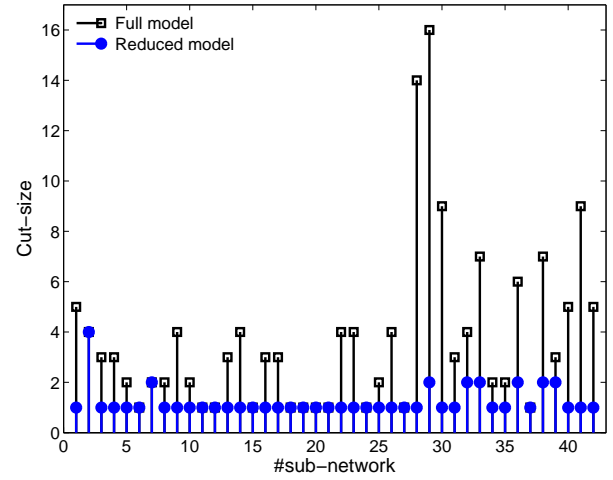

(a) Cut-size

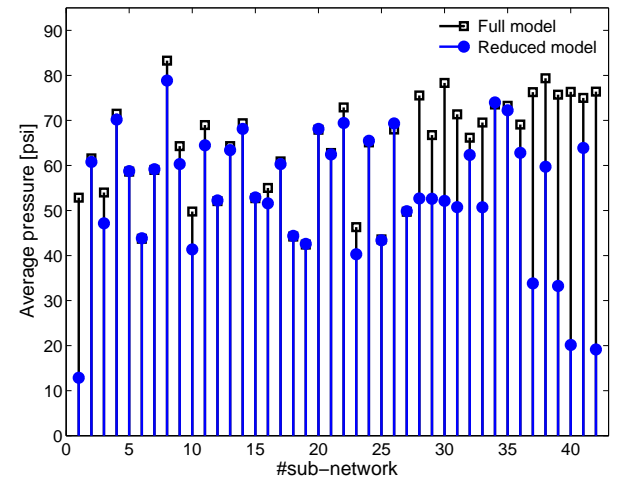

(b) Average pressure

Figure 3: EXNet sub-networks' performance: full model (black squares) and reduced model (blue fill rectangles)

pressures of each of the sub-networks for the full (black squares) and reduced (blue circles) models of EXNet based on the full hydraulic simulation. It can be observed that the cut size is significantly reduced after the optimization followed by a reduction in the average pressures in the system, although still above the minimum required. The average water age for each sub-network is reported in Table 2 in the SI, however, no apparent changes were observed between the full and reduced models for this network. Figure 3 in the SI demonstrates the pressure distribution in the network before (black-white) and after (blue) reconfiguration. As expected, the distribution of pressures is shifted to lower values after closing additional valves, since the energy losses in the system increase.

Figure 4 demonstrates similar analysis for BWSNII, although the number of boundary valves if greatly reduced after optimization (Figure 4a), there is only slight reduction in the average pressures for each sub-network (Figure 4b) and no apparent change in the water age. Figure 4 in the SI demonstrates the shift in the pressure distribution to lower values, similar to previous application.

Finally, Table 2 lists the different performance metrics explained previously. For both EDNet and BWSNII, we can observe the great reduction in the number of boundary connections, in terms of the total and the worst cut-size. This indicates the number of flow meters and pressure control valves that should be in installed in the inlet of each sub-network for 
water loss and pressure control on the network. A slight reduction is observed in both physical and complex network performance measures comparing the reduced and the full models. For BWSNII, the reduction in all measures is less significant than for the EXNet network, particularly the topological indicators, indicating that for large physical networks these measures are less informative.

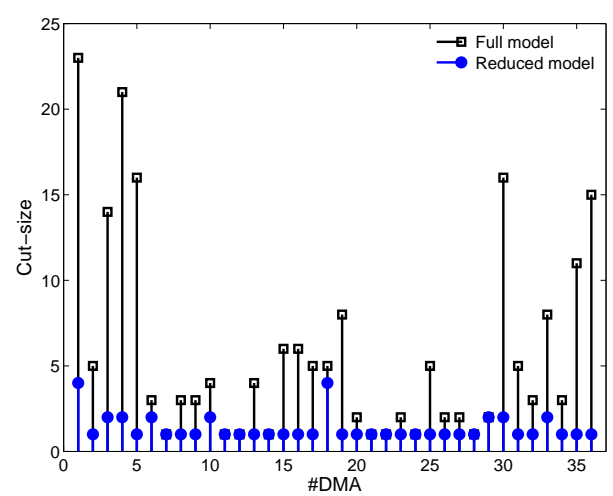

(a) Cut-size

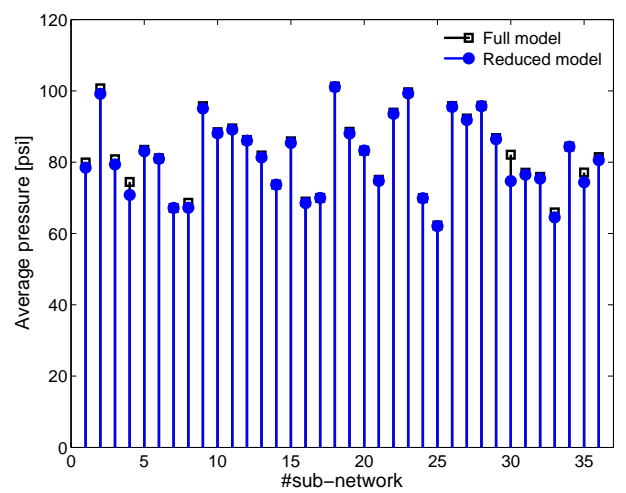

(b) Average pressure

Figure 4: BWSNII sub-networks' performance: full model (black squares) and reduced model (blue fill rectangles)

Table 2: Performance evaluation measures

\begin{tabular}{c|cc|cc} 
& \multicolumn{2}{|c|}{ EXNet } & \multicolumn{2}{c}{ BWSNII } \\
Metric & Full & Reduced & Full & Reduced \\
\hline $\boldsymbol{W} \boldsymbol{C} \boldsymbol{S}$ & 16 & 4 & 23 & 4 \\
$\boldsymbol{T} \boldsymbol{C} \boldsymbol{S}$ & 130 & 47 & 206 & 49 \\
$\boldsymbol{I}_{\boldsymbol{R}}$ & 0.72 & 0.64 & 0.98 & 0.96 \\
$\boldsymbol{I}_{\boldsymbol{N}}$ & 0.66 & 0.59 & 0.92 & 0.90 \\
$\boldsymbol{\lambda}_{\boldsymbol{2}}$ & 0.0004 & 0.0002 & -1.00 & -1.00 \\
$\boldsymbol{\Delta} \boldsymbol{\lambda}$ & 0.2612 & 0.2560 & 0.0062 & 0.0062 \\
$\boldsymbol{R}_{\boldsymbol{m}}$ & 0.1391 & 0.1172 & 0.0715 & 0.0652
\end{tabular}

In this paper, we introduce a practical and efficient approach for flexible water network reconfiguration facilitating water loss control and pressure management. In our approach, the network reconfiguration problem combines graph theory algorithms and is formulated as a LP problem, which is efficiently solved for large-scale networks. We examine the resiliency and robustness of different reconfiguration schemes based on common resiliency measures. Our results demonstrate the benefits and disadvantages from network decentralization. The 
presented approach provides a decision support tool for water utilities facilitating in infrastructure management.

\section{Acknowledgement}

The Authors thank the MIT-Technion Fellowship for supporting this research and Singapore Public Utility Board (PUB) for their collaboration.

\section{Supporting Information Available}

Auxiliary data providing the full networks data, related work, linear approximation, illustrative example for performance metrics, and results are provided in the SI.

This material is available free of charge via the Internet at http://pubs.acs.org/.

\section{References}

(1) Kingdom, B.; Liemberger, R.; Marin, P. The challenge of reducing non-revenue water (NRW) in developing countries; World Bank: Washington, DC, 2006.

(2) Thornton, J.; Sturm, R.; Kunkel, G. Water Loss Control; McGraw-Hill Companies, Inc., 2008.

(3) Kunkel, G. Committee report: Applying worldwide BMPs in water loss control. J. Am. Water Works Assoc. 2003, 95, 65-79.

(4) Morrison, J. Managing leakage by district meterd areas: a practical approach. Water21 2004, 6, 44-46.

(5) Ulanicki, B.; Meguid, H. A.; Bounds, P.; Patel, R. Pressure control in district metering areas with boundary and internal pressure reducing valves. Proc. 10th Water Distr. Syst. Anal. Conf. 2008; pp 691-703. 
(6) Ulanicki, B.; Bounds, P.; Rance, J.; Reynolds, L. Open and closed loop pressure control for leakage reduction. Urban Water 2000, 2, 105 - 114, Developments in water distribution systems.

(7) Wright, R.; Stoianov, I.; Parpas, P. Dynamic Topology in Water Distribution Networks. Procedia Engineering 2014, 70, 1735 - 1744, 12th International Conference on Computing and Control for the Water Industry, CCWI2013.

(8) Farley, M. Leakage management and control; World Health Organization: CH-1211 Geneva, Switzerland, 2001.

(9) Murray, R.; Grayman, W.; Savic, D.; Farmani, R. Effects of DMA redesign on water distribution system performance; Boxall and Maksimovi'c (eds): Taylor and Francis Group, London, 2010; pp 645-650.

(10) Ferrari, G.; Savic, D.; Becciu, G. A Graph Theoretic Approach and Sound Engineering Principles for Design of District Metered Areas. J. Water Resour. Plann. Manage. 2013,

(11) Deuerlein, J. W. Decomposition model of a general water supply network graph. J. Hydraul. Eng. 2008, 134, 822-832.

(12) Perelman, L.; Ostfeld, A. Topological clustering for water distribution systems analysis. Environ. Modell. Software 2011, 26, 969 - 972.

(13) Alvisi, S.; Franchini, M. A heuristic procedure for the automatic creation of district metered areas in water distribution systems. Urban Water Journal 2014, 11, 137-159.

(14) Diao, K.; Zhou, Y.; Rauch, W. Automated Creation of District Metered Area Boundaries in Water Distribution Systems. J. Water Resour. Plann. Manage. 2013, 139, 184-190. 
(15) DiNardo, A.; DiNatale, M.; Santonastaso, G. F.; Venticinque, S. An Automated Tool for Smart Water Network Partitioning. Water Resour. Manage. 2013, 27, 4493-4508.

(16) DiNardo, A.; DiNatale, M.; Santonastaso, G.; Tzatchkov, V.; Alcocer-Yamanaka, V. Water Network Sectorization Based on Graph Theory and Energy Performance Indices. J. Water Resour. Plann. Manage. 2013,

(17) USEPA, EPANET 2.00.12; U.S. Environmental Protection Agency: Cincinnati, Ohio, 2002; http: //www2 .epa.gov/water-research/epanet, Accessed: 2014-10-24.

(18) Giustolisi, O.; Savic, D.; Kapelan, Z. Pressure-Driven Demand and Leakage Simulation for Water Distribution Networks. Journal of Hydraulic Engineering 2008, 134, 626635.

(19) MOSEK ApS, D. The MOSEK optimization toolbox for MATLAB manual. Version 7.0. 2014; http://www.mosek.com/, Accessed: 2014-10-24.

(20) Gurobi Optimization, I. Gurobi Optimizer Reference Manual. 2014; http://www . gurobi.com, Accessed: 2014-10-24.

(21) Jolly, M.; Lothes, A.; Sebastian Bryson, L.; Ormsbee, L. Research Database of Water Distribution System Models. Journal of Water Resources Planning and Management 2014, 140, 410-416.

(22) Todini, E.; Rossman, L. Unified Framework for Deriving Simultaneous Equation Algorithms for Water Distribution Networks. J. Hydraul. Eng. 2013, 139, 511-526.

(23) Pohl, I. S. Bi-directional and Heuristic Search in Path Problems. Ph.D. thesis, 1969; AAI7001588.

(24) Karypis, G.; Kumar, V. Multilevel k-way Partitioning Scheme for Irregular Graphs. J. Parall. Distr. Comp. 1998, 48, 96-129. 
(25) METIS, version 5.1.0 University of Minnesota. 2013; http://glaros.dtc.umn.edu/ gkhome/metis/metis/download, Accessed: 2014-10-24.

(26) Sela Perelman, L.; Allen, M.; Preis, A.; Iqbal, M.; Whittle, A. J. Automated sub-zoning of water distribution systems. Environmental Modelling Software 2015, 65, 1 - 14 .

(27) Boulos, P. F.; Lansey, K. E.; Karney, B. W. Comprehensive water distribution systems analysis handbook for engineers and planners; MWH Soft, Inc. Publ.: Pasadena, CA, 2006.

(28) Gleixner, A. M.; Held, H.; Huang, W.; Vigerske, S. Towards globally optimal operation of water supply networks; 2012.

(29) Zhang, W.; Chung, G.; Pierre-Louis, P.; Bayraksan, G.; Lansey, K. Reclaimed water distribution network design under temporal and spatial growth and demand uncertainties. Environ. Modell. Software 2013, 49, 103-117.

(30) Salomons, E.; Goryashko, A.; Shamir, U.; Rao, Z.; Alvisi, S. Optimizing the operation of the Haifa-A water-distribution network. J. Hydroinf. 2007, 9, 51-64.

(31) Raad, D. N.; Sinske, A. N.; van Vuuren, J. H. Comparison of four reliability surrogate measures for water distribution systems design. Water Resour. Res. 2010, 46, W05524.

(32) Yazdani, A.; Jeffrey, P. Applying network theory to quanitfy the redundancy and structural robustness of water distribution systems. J. Water Resour. Plann. Manage. 2012, $138,153-161$.

(33) Todini, E. Looped water distribution networks design using a resilience index based heuristic approach. Urban Water 2000, 2, 115-122.

(34) Prasad, T. D.; Park, N. S. Multiobjective genetic algorithms for design of water distribution networks. J. Water Resour. Plann. Manage. 2004, 130, 73-82. 
(35) Buhl, J.; Gautrais, J.; Reeves, N.; Sol, R. V.; Valverde, S.; Kuntz, P.; Theraulaz, G. Topological patterns in street networks of self-organized urban settlements. The European Physical Journal B - Condensed Matter and Complex Systems 2006, 49, 513-522.

(36) Estrada, E. Network robustness to targeted attacks. The interplay of expansibility and degree distribution. The European Physical Journal B - Condensed Matter and Complex Systems 2006, 52, 563-574.

(37) Fiedler, M. Algebraic connectivity of graphs. Czechoslovak Mathematical Journal 1973, 23, 298-305.

(38) Alperovits, E.; Shamir, U. Design of optimal water distribution systems. Water Resour. Res. 1977, 13, 885-900.

(39) Wang, Q.; Guidolin, M.; Savic, D.; Kapelan, Z. Two-Objective Design of Benchmark Problems of a Water Distribution System via MOEAs: Towards the Best-Known Approximation of the True Pareto Front. Journal of Water Resources Planning and Management 2015, 141, 04014060 .

(40) Ostfeld, A. et al. The battle of the water sensor networks: a design challenge for engineers and algorithms. J. Water Resour. Plann. Manage. 2008, 134, 556-568. 
${ }_{441}$ Graphical TOC Entry

442 


\section{Flexible reconfiguration of existing urban water}

\section{infrastructure systems \\ Supporting Information}

Lina Sela Perelman, ${ }^{*, \dagger}$, Michael Allen, ${ }^{\ddagger}, \|$ Ami Preis, "Mudasser lqbal, " and Andrew J. Whittle ${ }^{\dagger, \perp}$

Department of Civil and Environmental Engineering, MIT, Cambridge, MA, USA, Faculty

of Engineering and Computing, Coventry University, UK, and Visenti ltd., Singapore

E-mail: linasela@mit.edu

${ }^{*}$ To whom correspondence should be addressed

${ }^{\dagger} \mathrm{MIT}$

${ }^{\ddagger}$ Coventry University

IVisenti

$\S$ Postdoctoral Fellow

"Research Fellow

${ }^{\perp}$ Edmund K. Turner Professor 
Table 1: Summary of methodologies for DMA design

\begin{tabular}{|c|c|c|c|}
\hline Paper & Design criteria & Solution method & Performance evaluation \\
\hline $\begin{array}{l}\text { Murray et al. } \\
(2010)\end{array}$ & $\begin{array}{l}\text { Connectedness to source } \\
\text { Adding/closing pipes } \\
\text { Size constraints }\end{array}$ & $\begin{array}{l}\text { Manual } \\
\text { DDA, EPANET }\end{array}$ & $\begin{array}{l}\text { Water security } \\
\text { Water age } \\
\text { Resilience index } \\
\text { Fire flow }\end{array}$ \\
\hline $\begin{array}{l}\text { Ferrari et al. } \\
(2013)\end{array}$ & $\begin{array}{l}\text { Connectedness to source } \\
\text { Closing pipes } \\
\text { Size constraints }\end{array}$ & $\begin{array}{l}\text { BFS } \\
\text { DDA, EPANET } \\
\text { Heuristics }\end{array}$ & Minimum pressure \\
\hline $\begin{array}{l}\text { Diao et al. } \\
(2013)\end{array}$ & $\begin{array}{l}\text { Closing pipes } \\
\text { Size constraints }\end{array}$ & $\begin{array}{l}\text { Modularity } \\
\text { DDA } \\
\text { Heuristics }\end{array}$ & $\begin{array}{l}\text { Minimum pressure } \\
\text { Water age } \\
\text { Fire flow }\end{array}$ \\
\hline $\begin{array}{l}\text { DiNardo et al. } \\
(2013)\end{array}$ & $\begin{array}{l}\text { Connectedness to source } \\
\text { Closing pipes }\end{array}$ & $\begin{array}{l}\text { DFS } \\
\text { PDA, WDNetXL } \\
\text { GA }\end{array}$ & $\begin{array}{l}\text { Resilience index } \\
\text { Pressure index } \\
\text { Flow index }\end{array}$ \\
\hline $\begin{array}{l}\text { DiNardo et al. } \\
(2013)\end{array}$ & $\begin{array}{l}\text { Closing pipes } \\
\text { Number of zones }\end{array}$ & $\begin{array}{l}\text { Graph partitioning } \\
\text { GA } \\
\text { DDA \& PDA }\end{array}$ & $\begin{array}{l}\text { Resilience index } \\
\text { Pressure index } \\
\text { Flow index }\end{array}$ \\
\hline $\begin{array}{l}\text { Alvisi and Franchini } \\
(2014)\end{array}$ & $\begin{array}{l}\text { Closing pipes } \\
\text { Size constraints }\end{array}$ & $\begin{array}{l}\text { BFS } \\
\text { DDA } \\
\text { Enumeration }\end{array}$ & $\begin{array}{l}\text { Minimum pressure } \\
\text { Resilience index }\end{array}$ \\
\hline This paper & $\begin{array}{l}\text { Connectedness to source } \\
\text { Closing valves } \\
\text { Size constraints } \\
\text { Minimum connections }\end{array}$ & $\begin{array}{l}\text { Graph partitioning } \\
\text { LP }\end{array}$ & $\begin{array}{l}\text { Hydraulic measures } \\
\text { Robustness metrics } \\
\text { Resilience indexes }\end{array}$ \\
\hline
\end{tabular}

BFS - Breadth first search; DFS - Depth first search; DDA - Demand driven analysis; PDA - Pressure driven analysis; GA - Genetic algorithms; LP - linear programming; 


\section{Physical surrogate measures}

Power loss in flow networks is defined by the summation over all links of the headloss $h_{j}$ multiplied by the flow $q_{j}$. It can be shown that an equivalent formulation is the summation over all nodes of the head $H_{i}$ multiplied by the nodal demand $b_{i}$, and formulated as:

$$
\sum_{j \in E} h_{j} q_{j}=h^{T} q=(A H)^{T} q=H^{T} A^{T} q=H^{T} d=\sum_{i \in N} H_{i} d_{i}
$$

where $A$ is network connectivity matrix.

The right hand side of (1) can be further decomposed into $P_{\text {in }}$, the power input by the network sources $N_{s}$, and $P_{\text {out }}$, the power output to network consumers $N_{d}$.

$$
\begin{gathered}
\sum_{j \in E} h_{j} q_{j}=\sum_{i \in N_{s}} H_{i} d_{i}-\sum_{i \in N_{d}} H_{i} d_{i} \\
\Downarrow \\
P_{\text {loss }}=P_{\text {in }}-P_{\text {out }}
\end{gathered}
$$



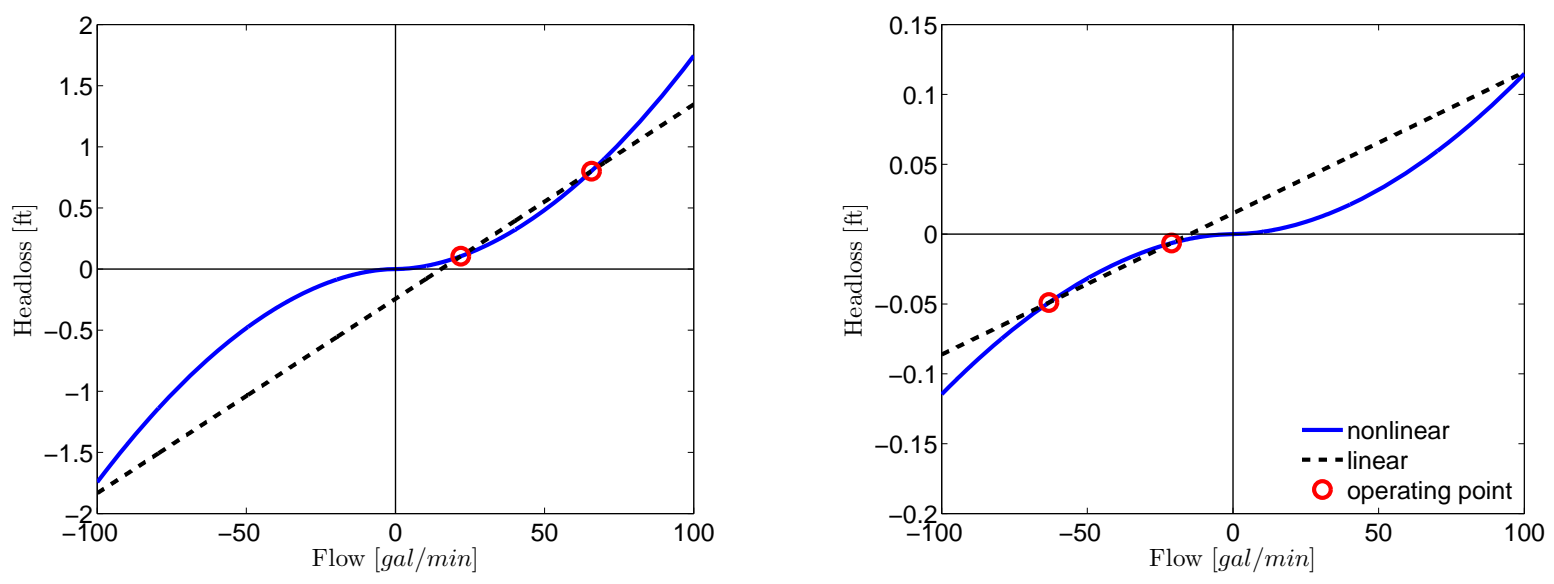

Figure 1: Linear approximation of the flow-headloss function. Figures a and b demonstrate linear approximation of the nonlinear flow-headloss function for pipes 2055 and 2056 of the EXNet network given an operating domain $\left[Q_{1}, Q_{2}\right]$. The operating domain is determined based on flows during normal operation. The linear model is computed as: $\tilde{h}(q)=\frac{h\left(Q_{2}\right)-h\left(Q_{1}\right)}{Q_{2}-Q_{1}} q+\frac{h\left(Q_{1}\right) Q_{2}-h\left(\left(Q_{2}\right) Q 1\right.}{Q_{2}-Q_{1}}=a_{1} q+a_{0}$. 


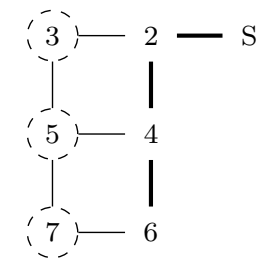

a1

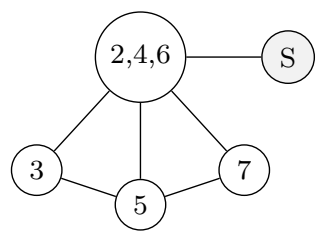

a2

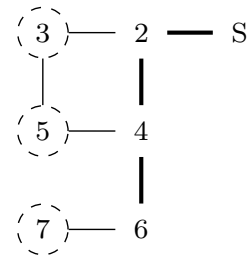

b1

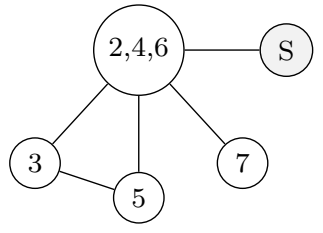

b2

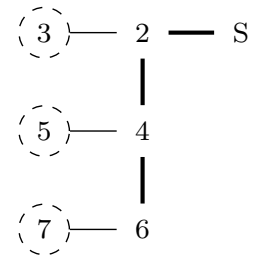

c1

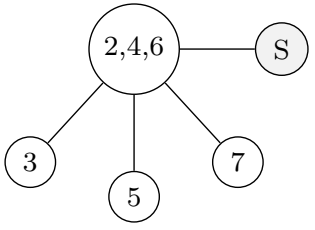

c2

\begin{tabular}{c|c|c|c} 
& $\mathbf{a}$ & $\mathbf{b}$ & $\mathbf{c}$ \\
\hline $\boldsymbol{W} \boldsymbol{C S}$ & 3 & 2 & 1 \\
$\boldsymbol{T} \boldsymbol{C S}$ & 5 & 4 & 3 \\
$\boldsymbol{P}_{\boldsymbol{m i n}}$ & 29.01 & 29.00 & 26.92 \\
$\boldsymbol{I}_{\boldsymbol{R}}$ & 0.927 & 0.927 & 0.914 \\
$\boldsymbol{I}_{\boldsymbol{N}}$ & 0.708 & 0.847 & 0.835 \\
$\boldsymbol{R}_{\boldsymbol{m}}$ & 0.222 & 0.111 & 0.000 \\
$\boldsymbol{\Delta}_{\boldsymbol{\lambda}}$ & 1.221 & 1.090 & 0.844 \\
$\boldsymbol{\lambda}_{\mathbf{2}}$ & 0.365 & 0.265 & 0.232 \\
\hline \multicolumn{3}{|l}{}
\end{tabular}

WCS - worst cut size; TCS - Total cut size; $P_{\min }$ - minimum pressure; $I_{R}$ - resiliency index;

$I_{N}$ - network resiliency index; $R_{m}$ - meshedness coefficient; $\Delta \lambda$ - spectral gap; $\lambda_{2}$ - algebraic connectivity;

Figure 2: Illustrative example - Network reconfiguration into a star-like topology and multicriteria performance metrics. Transmission mains are highlighted in subfigures a1-c1. The corresponding star-structure is shown in subfigures a2-c2, each time removing a boundary connection, i.e. closing the boundary valves. The table lists the measures for the three reconfigurations. 


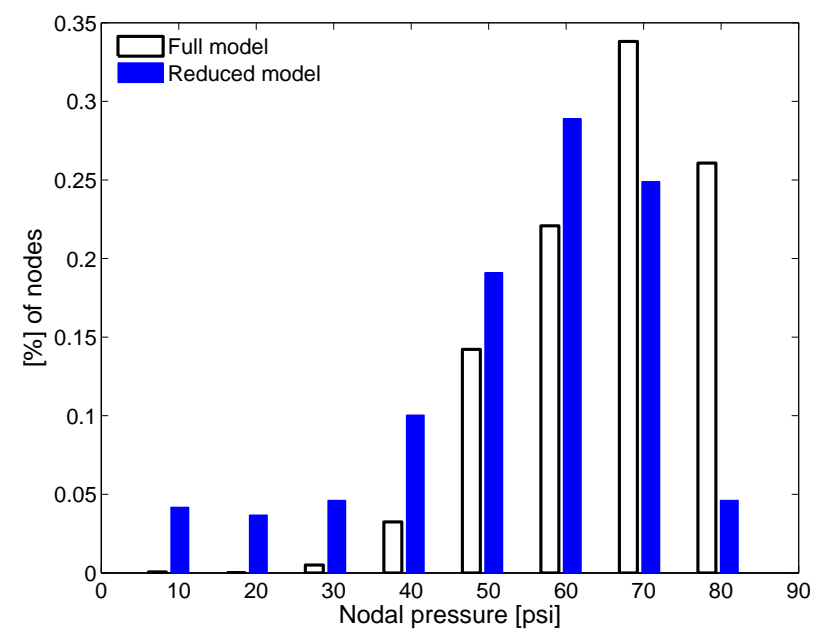

Figure 3: Pressure distribution in EXNet: full model (black-white) and reduced model after optimization (blue) 
Table 2: EXNet - sub-networks' data

\begin{tabular}{|c|c|c|c|c|c|c|}
\hline \multirow{2}{*}{$\begin{array}{c}\text { Sub- } \\
\text { network }\end{array}$} & \multirow{2}{*}{$\begin{array}{c}\text { Demand } \\
{\left[10^{5} \mathrm{gal} / \text { day }\right]}\end{array}$} & \multicolumn{2}{|c|}{ Full model } & \multicolumn{2}{|c|}{ Reduced model } & \multirow{2}{*}{$\begin{array}{l}\overline{W A} \\
{[h r]}\end{array}$} \\
\hline & & Cut-size & $\bar{P}[p s i]$ & Cut-size & $\bar{P}[p s i]$ & \\
\hline main & 114.53 & 103 & 63.03 & 42 & 61.08 & 10.41 \\
\hline 2 & 14.34 & 5 & 52.85 & 1 & 12.88 & 3.85 \\
\hline 3 & 11.33 & 4 & 61.57 & 4 & 60.79 & 11.47 \\
\hline 4 & 6.82 & 3 & 54.00 & 1 & 47.16 & 9.05 \\
\hline 5 & 5.76 & 3 & 71.48 & 1 & 70.20 & 10.72 \\
\hline 6 & 5.74 & 2 & 58.64 & 1 & 58.74 & 8.82 \\
\hline 7 & 4.98 & 1 & 43.71 & 1 & 43.84 & 6.99 \\
\hline 8 & 4.59 & 2 & 59.02 & 2 & 59.18 & 9.27 \\
\hline 9 & 4.23 & 2 & 83.28 & 1 & 78.85 & 14.80 \\
\hline 10 & 3.95 & 4 & 64.29 & 1 & 60.31 & 14.18 \\
\hline 11 & 3.87 & 2 & 49.78 & 1 & 41.36 & 11.06 \\
\hline 12 & 3.64 & 1 & 68.97 & 1 & 64.47 & 13.38 \\
\hline 13 & 3.58 & 1 & 52.08 & 1 & 52.25 & 10.63 \\
\hline 14 & 3.45 & 3 & 64.29 & 1 & 63.39 & 9.65 \\
\hline 15 & 3.42 & 4 & 69.38 & 1 & 68.11 & 13.33 \\
\hline 16 & 3.39 & 1 & 52.75 & 1 & 52.91 & 9.31 \\
\hline 17 & 3.28 & 3 & 55.01 & 1 & 51.59 & 9.08 \\
\hline 18 & 2.91 & 3 & 60.91 & 1 & 60.29 & 7.97 \\
\hline 19 & 2.85 & 1 & 44.22 & 1 & 44.38 & 6.91 \\
\hline 20 & 2.84 & 1 & 42.45 & 1 & 42.60 & 8.85 \\
\hline 21 & 2.80 & 1 & 67.95 & 1 & 68.13 & 10.46 \\
\hline 22 & 2.77 & 1 & 62.76 & 1 & 62.45 & 13.22 \\
\hline 23 & 2.70 & 4 & 72.88 & 1 & 69.42 & 11.79 \\
\hline 24 & 2.66 & 4 & 46.31 & 1 & 40.28 & 5.40 \\
\hline 25 & 2.59 & 1 & 65.12 & 1 & 65.49 & 10.43 \\
\hline 26 & 2.52 & 2 & 43.58 & 1 & 43.40 & 7.05 \\
\hline 27 & 2.08 & 4 & 67.99 & 1 & 69.34 & 12.67 \\
\hline 28 & 1.60 & 1 & 49.72 & 1 & 49.83 & 4.08 \\
\hline 29 & 21.71 & 14 & 75.53 & 1 & 52.67 & 13.39 \\
\hline 30 & 11.06 & 16 & 66.74 & 2 & 52.60 & 11.80 \\
\hline 31 & 14.96 & 9 & 78.33 & 1 & 52.14 & 13.48 \\
\hline 32 & 1.40 & 3 & 71.35 & 1 & 50.75 & 10.92 \\
\hline 33 & 6.68 & 4 & 66.16 & 2 & 62.34 & 13.81 \\
\hline 34 & 10.85 & 7 & 69.54 & 2 & 50.71 & 12.40 \\
\hline 35 & 4.64 & 2 & 73.55 & 1 & 74.00 & 12.62 \\
\hline 36 & 4.58 & 2 & 73.25 & 1 & 72.26 & 11.88 \\
\hline 37 & 7.03 & 6 & 69.09 & 2 & 62.82 & 13.03 \\
\hline 38 & 2.06 & 1 & 76.27 & 1 & 33.81 & 16.26 \\
\hline 39 & 17.35 & 7 & 79.37 & 2 & 59.72 & 14.90 \\
\hline 40 & 10.55 & 3 & 75.72 & 2 & 33.24 & 17.34 \\
\hline 41 & 13.54 & 5 & 76.35 & 1 & 20.16 & 13.35 \\
\hline 42 & 11.13 & 9 & 75.00 & 1 & 63.89 & 13.16 \\
\hline 43 & 3.65 & 5 & 76.40 & 1 & 19.14 & 14.02 \\
\hline
\end{tabular}


Table 3: EXNet - boundary valves at final solution

\begin{tabular}{|c|c|c|}
\hline $\begin{array}{l}\text { Valve } \\
\text { ID }\end{array}$ & $\begin{array}{c}\text { Start } \\
\text { sub-network }\end{array}$ & $\begin{array}{c}\text { End } \\
\text { sub-network }\end{array}$ \\
\hline 5164 & 1 & 2 \\
\hline 2187 & 1 & 3 \\
\hline 2269 & 1 & 3 \\
\hline 2283 & 1 & 3 \\
\hline 2599 & 1 & 3 \\
\hline 4913 & 1 & 4 \\
\hline 3532 & 1 & 5 \\
\hline 3783 & 1 & 6 \\
\hline 5132 & 1 & 7 \\
\hline 3593 & 1 & 8 \\
\hline 2271 & 1 & 8 \\
\hline 2605 & 1 & 9 \\
\hline 2760 & 1 & 10 \\
\hline 4878 & 1 & 11 \\
\hline 2197 & 1 & 12 \\
\hline 2424 & 1 & 13 \\
\hline 2313 & 1 & 14 \\
\hline 3634 & 1 & 15 \\
\hline 2122 & 1 & 16 \\
\hline 3859 & 1 & 17 \\
\hline 5076 & 1 & 18 \\
\hline 5153 & 1 & 19 \\
\hline 3500 & 1 & 20 \\
\hline 2397 & 1 & 21 \\
\hline 2298 & 1 & 22 \\
\hline 4015 & 1 & 23 \\
\hline 4156 & 1 & 24 \\
\hline 5220 & 1 & 25 \\
\hline 3213 & 1 & 26 \\
\hline 2364 & 1 & 27 \\
\hline 2939 & 1 & 28 \\
\hline 4875 & 1 & 30 \\
\hline 2806 & 1 & 31 \\
\hline 3021 & 1 & 33 \\
\hline 2217 & 1 & 33 \\
\hline 5004 & 30 & 34 \\
\hline 3129 & 32 & 34 \\
\hline 3853 & 1 & 35 \\
\hline 2256 & 1 & 36 \\
\hline 2600 & 1 & 37 \\
\hline 2679 & 29 & 37 \\
\hline 2407 & 1 & 39 \\
\hline 4194 & 38 & 40 \\
\hline 5305 & 39 & 40 \\
\hline 5089 & 1 & 41 \\
\hline 2945 & 1 & 42 \\
\hline 2182 & 1 & 43 \\
\hline
\end{tabular}




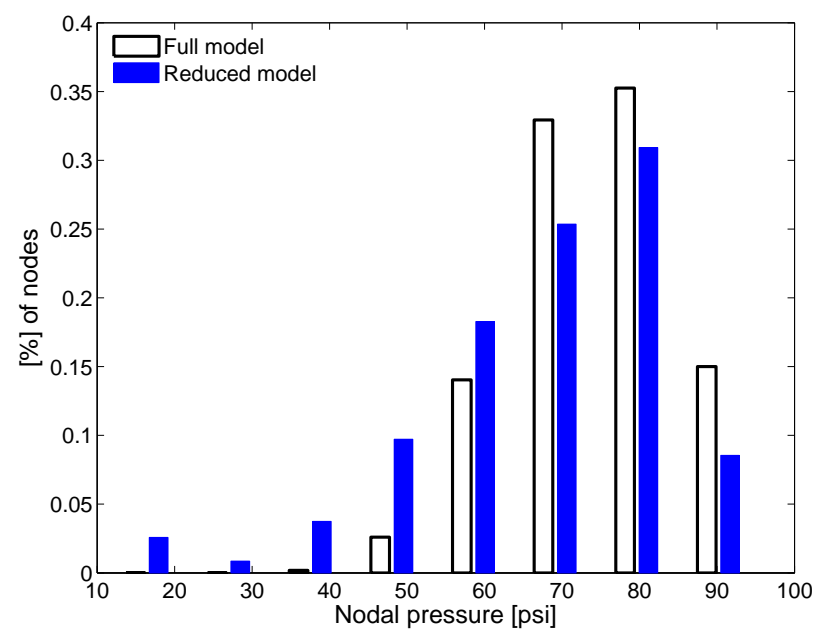

Figure 4: Pressure distribution in BWSNII: full model (black-white) and feduced model after optimization (blue) 
Table 4: BWSNII - sub-networks' data

\begin{tabular}{|c|c|c|c|c|c|c|}
\hline \multirow{2}{*}{$\begin{array}{c}\text { Sub- } \\
\text { network }\end{array}$} & \multirow{2}{*}{$\begin{array}{c}\text { Demand } \\
{\left[10^{5} \mathrm{gal} / \text { day }\right]}\end{array}$} & \multicolumn{2}{|c|}{ Full model } & \multicolumn{2}{|c|}{ Reduced model } & \multirow{2}{*}{$\begin{array}{l}\overline{W A} \\
{[h r]}\end{array}$} \\
\hline & & Cut-size & $\bar{P}[p s i]$ & Cut-size & $\bar{P}[p s i]$ & \\
\hline main & 110.30 & 202 & 82.12 & 49 & 81.82 & 15.00 \\
\hline 2 & 14.47 & 23 & 79.91 & 4 & 78.46 & 9.41 \\
\hline 3 & 9.18 & 5 & 100.70 & 1 & 99.17 & 21.17 \\
\hline 4 & 8.29 & 14 & 80.83 & 2 & 79.37 & 13.31 \\
\hline 5 & 7.64 & 21 & 74.46 & 2 & 70.82 & 12.78 \\
\hline 6 & 4.82 & 16 & 83.47 & 1 & 83.05 & 22.47 \\
\hline 7 & 4.73 & 3 & 81.09 & 2 & 80.95 & 22.63 \\
\hline 8 & 4.61 & 1 & 67.17 & 1 & 67.16 & 7.55 \\
\hline 9 & 4.55 & 3 & 68.60 & 1 & 67.22 & 11.24 \\
\hline 10 & 4.49 & 3 & 95.70 & 1 & 95.05 & 22.69 \\
\hline 11 & 4.01 & 4 & 88.40 & 2 & 88.08 & 16.43 \\
\hline 12 & 3.27 & 1 & 89.46 & 1 & 89.15 & 14.59 \\
\hline 13 & 2.70 & 1 & 86.21 & 1 & 86.06 & 14.85 \\
\hline 14 & 2.56 & 4 & 81.87 & 1 & 81.32 & 13.14 \\
\hline 15 & 2.49 & 1 & 73.71 & 1 & 73.70 & 17.53 \\
\hline 16 & 2.32 & 6 & 85.84 & 1 & 85.38 & 9.31 \\
\hline 17 & 2.04 & 6 & 68.94 & 1 & 68.52 & 14.31 \\
\hline 18 & 2.02 & 5 & 69.97 & 1 & 69.96 & 7.51 \\
\hline 19 & 2.01 & 5 & 101.21 & 4 & 101.06 & 23.17 \\
\hline 20 & 1.80 & 8 & 88.51 & 1 & 88.03 & 22.55 \\
\hline 21 & 1.72 & 2 & 83.22 & 1 & 83.25 & 6.40 \\
\hline 22 & 1.41 & 1 & 75.06 & 1 & 74.75 & 14.94 \\
\hline 23 & 1.40 & 1 & 93.88 & 1 & 93.55 & 22.20 \\
\hline 24 & 1.18 & 2 & 99.58 & 1 & 99.29 & 22.04 \\
\hline 25 & 1.18 & 1 & 69.95 & 1 & 69.88 & 9.37 \\
\hline 26 & 1.07 & 5 & 62.25 & 1 & 62.07 & 23.64 \\
\hline 27 & 1.05 & 2 & 95.73 & 1 & 95.50 & 23.07 \\
\hline 28 & 1.02 & 2 & 92.23 & 1 & 91.80 & 22.06 \\
\hline 29 & 1.01 & 1 & 95.85 & 1 & 95.70 & 18.05 \\
\hline 30 & 1.01 & 2 & 86.75 & 2 & 86.45 & 20.26 \\
\hline 31 & 12.43 & 16 & 82.09 & 2 & 74.69 & 10.72 \\
\hline 32 & 12.11 & 5 & 77.05 & 1 & 76.48 & 6.11 \\
\hline 33 & 8.08 & 3 & 75.88 & 1 & 75.38 & 16.01 \\
\hline 34 & 12.71 & 8 & 65.90 & 2 & 64.52 & 10.04 \\
\hline 35 & 10.10 & 3 & 84.38 & 1 & 84.35 & 6.49 \\
\hline 36 & 13.52 & 11 & 77.11 & 1 & 74.38 & 19.96 \\
\hline 37 & 2.94 & 15 & 81.41 & 1 & 80.57 & 15.38 \\
\hline
\end{tabular}


Table 5: BWSNII - boundary valves at final solution

\begin{tabular}{|c|c|c|}
\hline $\begin{array}{l}\text { Valve } \\
\text { ID }\end{array}$ & $\begin{array}{c}\text { Start } \\
\text { sub-network }\end{array}$ & $\begin{array}{c}\text { End } \\
\text { sub-network }\end{array}$ \\
\hline LINK-200 & 1 & 2 \\
\hline LINK-6446 & 1 & 2 \\
\hline LINK-9623 & 1 & 2 \\
\hline LINK-13127 & 1 & 2 \\
\hline LINK-2848 & 1 & 3 \\
\hline LINK-1385 & 1 & 4 \\
\hline LINK-1783 & 1 & 4 \\
\hline LINK-13867 & 1 & 5 \\
\hline LINK-13955 & 1 & 5 \\
\hline LINK-10321 & 1 & 6 \\
\hline LINK-11131 & 1 & 7 \\
\hline LINK-11137 & 1 & 7 \\
\hline LINK-7355 & 1 & 8 \\
\hline LINK-11433 & 1 & 9 \\
\hline LINK-320 & 1 & 10 \\
\hline LINK-6107 & 1 & 11 \\
\hline LINK-7218 & 1 & 11 \\
\hline LINK-6411 & 1 & 12 \\
\hline LINK-3939 & 1 & 13 \\
\hline LINK-5124 & 1 & 14 \\
\hline LINK-9195 & 1 & 15 \\
\hline LINK-6750 & 1 & 16 \\
\hline LINK-14278 & 1 & 17 \\
\hline LINK-8131 & 1 & 18 \\
\hline LINK-4374 & 1 & 19 \\
\hline LINK-4542 & 1 & 19 \\
\hline LINK-5783 & 1 & 19 \\
\hline LINK-10904 & 1 & 19 \\
\hline LINK-3705 & 1 & 20 \\
\hline LINK-9248 & 1 & 21 \\
\hline LINK-5276 & 1 & 22 \\
\hline LINK-6342 & 1 & 23 \\
\hline LINK-784 & 1 & 24 \\
\hline LINK-9581 & 1 & 25 \\
\hline LINK-4194 & 1 & 26 \\
\hline LINK-10795 & 1 & 27 \\
\hline LINK-5835 & 1 & 28 \\
\hline LINK-12170 & 1 & 29 \\
\hline LINK-6464 & 1 & 30 \\
\hline LINK-6845 & 1 & 30 \\
\hline LINK-978 & 1 & 31 \\
\hline LINK-14243 & 1 & 31 \\
\hline LINK-7723 & 1 & 32 \\
\hline LINK-14516 & 1 & 33 \\
\hline LINK-8072 & 1 & 34 \\
\hline LINK-8406 & 1 & 34 \\
\hline LINK-9228 & 1 & 35 \\
\hline LINK-13681 & 1 & 36 \\
\hline LINK-6891 & 1 & 37 \\
\hline
\end{tabular}

